\title{
Pregnancy in a Global Pandemic: Primum non nocere - First do no harm
}

\author{
Alexandra Kermack ${ }^{1}$, Linden Stocker ${ }^{1}$, Ying Cheong ${ }^{1}$, and Simon Crawford ${ }^{2}$ \\ ${ }^{1}$ University of Southampton Faculty of Medicine \\ ${ }^{2}$ University Hospital Southampton NHS Foundation Trust
}

April 28, 2020

As clinicians, we are familiar with the Hippocratic albatross 'Primum non nocere', 'first, do not harm'. We understand that every weapon in our clinical armour chest is double-edged, and every cure has a potential harm, this has never more true than during the global pandemic of SARS-CoV-2.

In these unprecedented times, the UK government acted swiftly to try to first contain the virus and then flatten the peak of admissions to hospital. On $16^{\text {th }}$ March, pregnant women were placed in the most vulnerable, 'at risk', category advising them to self-isolate. This message has been carefully observed by women. Whilst the press 'floods' us with images of overwhelmed hospitals, the normal patient throughput in obstetrics and gynaecology to semi-urgent care is anecdotally at an all-time low. Women with pain and bleeding, in early pregnancy, are staying away from hospital. Antenatal women are not presenting with, 'threatened', labour nor reduced fetal movements. The true incidence of these conditions has not simply declined. Certainly, some of these women are not presenting and are suffering at home.

According to NHS England figures, there was a $29 \%$ drop in numbers of people attending Accident and Emergency departments in March 2020 -1.5 million compared with 2.2 million in March 2019 (NHS England figures). The Royal College of Midwives became concerned patients were not attending scheduled appointments for pregnancy care, this led to a joint statement with the Royal College of Obstetricians and Gynaecologists (RCOG) emphasising the importance of antenatal care. There is concern that women may be self-isolating at home to their detriment. If they do develop medical problems, they may defer contacting medical professionals until symptoms are severe, and in some cases life-threatening, for example in ectopic pregnancies.

In order to limit hospital-footfall, women are being asked to attend alone. In normal circumstances, partners provide-support during antenatal ultrasounds or consultations, a time which some may receive upsetting news. Additionally, with centralisation of services to depleted staff numbers, there is less choice in the location of pregnancy-related care and birthplace. Important appointments are being missed, and in obstetrics, 'the clock' does not stop because of Covid-19.

A wider part of the role of the obstetrician is safeguarding. Children are out of school and domestic violence prevalent ${ }^{1}$. We are missing opportunities to identify vulnerable women, and to intervene for the safety and wellbeing of her and her children. Much emotional and practical support is now also lacking. Isolation to prevent coronavirus spread means grandparents cannot visit new babies, families struggle with the provision of childcare for existing children. The networking women partake in at antenatal classes, nor the support from visiting postnatal midwives nor breastfeeding support is happening. This, combined with the anxiety and fear felt during a global pandemic ${ }^{2}$, may be associated with a resurgence in postnatal mental health disorders. 
Despite this difficult time, there are positives. Patient care has been rapidly remodelled across the country with services streamlined in record time. For example, 'one stop', antenatal booking clinics encompass ultrasound, venepuncture and a face-to-face midwife consult. Previously this necessitated multiple appointments, with impacts on work and family life. Like most areas of medicine, clinics are being performed via telephone or online conference calls. This is time and cost-saving for both parties. The necessity or validity of investigations is being questioned more ruthlessly, perhaps as we should be doing routinely.

With reduced patient numbers, women in early pregnancy are being triaged and seen faster. The number of invasive surgical procedures performed for miscarriage and ectopic pregnancies has plummeted (University Hospital Southampton data 2020). Partly because women are encouraged to have less invasive management, thereby circumventing surgical risk. However, fewer ultrasounds are being performed for vague symptoms. Resulting in fewer women having, 'pregnancies of unknown location', a diagnosis fraught with iatrogenic anxiety and distress. Women are feeling more empowered to self-manage conditions that we have always advocated self-care for in the first instance. Fewer women are requesting induction of labour, usually a huge burden of work on innundated labour wards.

By responding to the global pandemic, we are likely causing harm to some of the women we have a duty of care for, we plan to look at national data to assess the level of secondary harm caused. The ameliorating benefits are that the NHS is being treated as a precious commodity, it is imperative that innovative changes and optimal uses of services bring about long term benefit. The UK healthcare system will change forever as a result of Covid-19; it is the responsibility of doctors and patients to ensure that this is for the better and to minimise harm to women and their families in the meantime.

\section{Contribution to authorship}

The idea was conceived by SC. All authors researched and compiled the manuscript.

\section{Disclosure of interests}

None of the authors have any conflicts of interest to declare.

\section{Ethics and funding}

No ethical approval or funding was required for the manuscript.

1. Bradbury-Jones C, Isham L. The pandemic paradox: the consequences of COVID-19 on domestic violence. J Clin Nurs. 2020 Apr 12.

2. Chew QH, Wei KC, Vasoo S, Chua HC, Sim K. Narrative synthesis of psychological and coping responses towards emerging infectious disease outbreaks in the general population: practical considerations for the COVID-19 pandemic. Singapore Med J. 2020 Apr 3. 